\title{
El diseño normativo de las pruebas periciales, a propósito del razonamiento inferencial de los expertos y la comprensión judicial
}

\author{
The Institutional Design of Expert Evidence. \\ Remarks on the Inferential Reasoning of Experts \\ and Judicial Comprehension
}

Carmen Vázquez

Resumen: La valoración judicial del conocimiento experto proveniente de una prueba pericial exige que los jueces comprendan el razonamiento inferencial en juego. Ello supone que los jueces controlen tanto las premisas del razonamiento pericial como las inferencias que se hacen a partir de ellas. Toda esta tarea debe realizarse básicamente en la práctica y en la admisión de las pruebas periciales, atendiendo tanto el fundamento de las generalizaciones expertas como a su aplicación al caso concreto. En este escenario, el objetivo será ofrecer a los operadores jurídicos y a los expertos diversas herramientas procesales para construir una práctica dialógica que permita lograr las exigencias que deben satisfacer sobre todo los jueces.

Palabras clave: Prueba pericial, razonamiento probatorio, desacuerdos entre expertos, diseño institucional.

Doctora en Derecho y Profesora. Universitat de Girona, Gerona, España. Correo electrónico: carmen.vazquez@udg.edu. Agradezco todos los valiosos comentarios que a una versión preliminar me hicieron Edgar Aguilera, Diego Dei Vecchi, Jordi Ferrer Beltrán y Carlo Vittorio Giabardo. También agradezco los útiles comentarios de los dos evaluadores anónimos. Para la realización de este trabajo he contado con el apoyo del proyecto de investigación "Seguridad Jurídica y razonamiento judicial” (DER2017-82661-P), del Ministerio español de Economía y Competitividad. 


\begin{abstract}
The judicial assessment of expert knowledge underlying expert evidence requires judges' comprehension of the inferential reasoning implied. This presupposes that judges should control both the premises of the expert reasoning and the inferences drawn from them. This whole task should be done during the admissibility stage and in the course of the presentation of the evidence during the trial, looking for the basis of experts' generalizations and assessing how they are applied to the specific case. In this scenario, the aim is to provide some tools in order for legal practitioners and experts to build a dialogic practice allowing both of them to fulfill their obligations, especially those of the judges.
\end{abstract}

Keywords: Expert evidence, evidential reasoning, disagreements between experts, institutional design.

\title{
1. Introducción
}

La valoración de la prueba pericial supone que los jueces deben comprender el razonamiento inferencial realizado por los peritos si están interesados, como deberían estarlo, en tomar decisiones racionales con él. Ello supone identificar las premisas usadas por los expertos para atender a sus fundamentos y las diversas inferencias realizadas a partir de ellas. Desafortunadamente, en los sistemas jurídicos, quizá sobre todo en los de tradición romano-germánica, no se ha delimitado claramente en qué consiste la valoración judicial de las pruebas periciales y, por supuesto, en general no se han desarrollado herramientas procesales con el objetivo de ayudar a los jueces a realizar su tarea (o, pese a su previsión en el ordenamiento, no han sido usadas para lo que deberían ser usadas). En su lugar, se ha incentivado de diversas maneras y distintos grados cierta deferencia hacia los peritos por el hecho de ser tenidos como expertos y, acorde con ello, se han buscado muy diversos criterios, que funcionarían como atajos, para supuestamente justificar el uso de conocimiento experto en las decisiones judiciales sin necesidad de que el juez comprenda el razonamiento inferencial de los peritos. Así tenemos criterios relacionados con el sujeto mismo, como la imparcialidad pericial; con el informe pericial, como su completitud o ausencia de contradicciones; o malos criterios sobre los fundamentos 
El diseño normativo de las pruebas periciales, a propósito del razonamiento...

de lo que afirma un experto, como la cientificidad del conocimiento; y un largo etcétera. ${ }^{1}$

Los atajos antes mencionados tienen diversos problemas en sí mismos. Por ejemplo, si consideramos la imparcialidad pericial en atención al origen de los expertos, es decir, básicamente a su nombramiento por parte de un juez y no por las partes, algunas veces pareciera que más bien estamos definiendo qué entenderemos por “imparcialidad pericial”. Esto es, un perito será considerado imparcial cuando ha sido designado de alguna manera por el juez; sin embargo, esta suerte de estipulación, obviamente, nada puede decir sobre la calidad de aquello que dice y hace un experto en un proceso judicial. Pero, lo que es peor, por más imparcial que un experto pudiera ser, todavía podría estar utilizando métodos o técnicas no fiables o con grandes rangos de error; un perito imparcial podría estar usando información incompleta sobre el caso; un perito imparcial, equivocarse en un caso concreto, etc. Por ello, la justificación de las premisas usadas por los expertos en su razonamiento tiene que buscarse fuera del sujeto (de su experiencia, de sus calificaciones o su pertenencia a una institución).

En los últimos años seguramente se ha dado un paso en la búsqueda de información externa al experto para justificar el uso de conocimiento experto, así algunas decisiones judiciales y/o estudiosos en la materia han acudido a la cientificidad de los métodos, técnicas, afirmaciones, etc., que usan o hacen los peritos. Sin embargo, las discusiones que en la filosofía de la ciencia ha habido sobre el supuesto carácter científico de algo, conocido como el problema de la demarcación, han mostrado la insuficiencia de los diversos criterios de cientificidad que se desarrollaron para delimitar claramente lo que sería científico y lo que no sería científico. Además de ello, no todo lo científico es igualmente fiable y no solo lo científico es fiable, por lo que tampoco podemos asumir que cientificidad es igual a fiabilidad. En cambio, para usar en las decisiones judiciales información experta de forma justificada necesitamos precisamente información sobre su fiabilidad, tanto sobre las generalizaciones usadas como en su aplicación al caso concreto en que se aplican.

1 No es este el espacio para profundizar en estas cuestiones, lo he hecho ya en diferentes trabajos (véase Vázquez, 2016, pp. 92-112; 2018, pp. 69-107). 
Es evidente que no basta solo con tener información sobre las premisas implicadas en el razonamiento pericial y sus fundamentos, el juez debe comprender lo que está en juego en el contexto del caso específico que está resolviendo. Es claro que el sistema jurídico solo puede poner un deber cuando este puede ser cumplido (deber implica poder) y, bajo esa consideración, se podría argumentar que los jueces no pueden cumplir con dicha obligación. Una de las explicaciones que se han dado al respecto, muy generalizada en la comunidad jurídica, es que los jueces, al ser legos en el conocimiento experto que subyace a las pruebas periciales, son incapaces de comprender las afirmaciones de los peritos. ${ }^{2}$ Tal asunción debería ser tomada muy en serio a la hora de construir nuestros diseños normativos, pues de ser cierta debería suponer un cambio bastante radical en la estructura actual del proceso judicial a efectos de retirar a los incapaces de tomar decisiones que indudablemente afectan a los ciudadanos. Evidentemente tales incapacidades serían una suerte de déficits cognitivos insalvables o difícilmente salvables, no déficits de información que pudieran ser ocasionados por falta de datos relevantes o incluso por desbordes de información.

Sin embargo, parece que cuando se han realizado estudios empíricos sobre la capacidad de los decisores sobre los hechos para valorar las pruebas periciales, los resultados contrastan con la asunción de una incapacidad cognitiva y más bien muestran una y otra vez serios déficits de información por parte de los decisores. En los casos ordinarios, algunas veces la información ausente es cultural, más general y atinente a la concepción que se tiene sobre el funcionamiento del conocimiento experto o de la empresa científica en particular; en algunas otras ocasiones la información ausente es sobre los hechos del caso y la ausencia es generada por ciertas malas prácticas y/o reglas deficientes. Quizá otra es la situación cuando se trata de casos especialmente complejos, donde sería esperable un aumento de las dificultades de comprensión de un juez sobre el conocimiento experto relevante para aquellos, en ese contexto deberíamos preguntarnos si el sistema en que tiene lugar ayuda (o no) para que el juez logre la comprensión de aquello que tiene que resolver.

2 Ello, por supuesto, a la vez que se afirma que el juez es "perito peritorum" y que de ninguna manera la prueba pericial es vinculante para los jueces. 
El diseño normativo de las pruebas periciales, a propósito del razonamiento...

En mi opinión, cuando se cuestionan las capacidades cognitivas de los jueces para tratar con las pruebas periciales, no solo hay que mostrar empíricamente tal hecho sino también en qué medida influye en ello el sistema jurídico en el que los jueces llevan a cabo su función.

Y, si lo anterior es así, entonces, el sistema jurídico debe ofrecer a sus jueces las herramientas necesarias para reducir o eliminar en la medida de lo posible sus déficits de información, preocupándose por asegurar la completitud de los datos desde la admisibilidad de las pruebas periciales hasta el empleo de diversos mecanismos dialógicos con los expertos que participan. El objetivo fundamental de este trabajo será entonces discutir el diseño institucional de un modelo con esos mimbres, bien para tenerlos como propuesta de lege ferenda o, en caso de que ya estén previstos, brindar ciertas pautas para su funcionamiento adecuado dado el objetivo de lograr la comprensión judicial. Para ello, en primer lugar, intentaré argumentar que necesitamos diseñar ordenamientos que reflejen un modelo de confianza hacia los jueces, no esparcir una sistemática desconfianza hacia ellos, sobre todo considerando la evidencia empírica que al respecto se tiene y la ausencia de alternativas serias.

En segundo lugar, desarrollaré un conjunto de diversos posibles mecanismos para incentivar la comprensión de los jueces fundamentalmente durante la práctica de las pruebas periciales: preguntas de aclaración durante el contradictorio, asesores expertos para casos complejos, meta-periciales para complementar las pruebas periciales inicialmente presentadas y los careos entre expertos para afrontar los desacuerdos entre los mismos. Por supuesto, garantizando en todo momento una adecuada participación de las partes en la práctica de cada una de tales herramientas.

Debo advertir desde ya que se trata de mecanismos que están individualmente previstos en diversos ordenamientos, tanto del common law como de tradición romano-germánica, en ese sentido mi propuesta no es original. Sin embargo, la originalidad radicaría en: (i) la propuesta de su conjunción en un mismo ordenamiento para incentivar y lograr la comprensión judicial del razonamiento pericial; y (ii) su aplicación en la práctica de las pruebas periciales durante el juicio oral, y no a efectos de la conformación de una prueba pericial o para decidir su admisibilidad.

Ahora bien, quizá una buena manera de incluso evitar el uso de los mecanismos antes planteados, sea prestar mayor atención a qué se pide a 
los expertos y qué información relevante se les brinda para que realicen la función que se les ha asignado. Por ello, en tercer lugar, defenderé que debe haber una mayor preocupación en la determinación del objeto del peritaje, de los datos relevantes que se le brindan al experto para la realización de sus operaciones periciales y en la información que este brinda sobre la fiabilidad de las generalizaciones independientes a los hechos del caso que utiliza.

Los argumentos y las posibles herramientas que aquí se presentan pretenden abonar a una concepción general de la prueba pericial, sin pretender siquiera sugerir que con ellos se terminarán los muy diversos problemas que se afrontan con los distintos tipos de pruebas periciales en los sistemas jurídicos. Por supuesto, cada país afronta problemas distintos con el conocimiento experto que es utilizado por sus tribunales como elemento de juicio: en algunos países el problema es que no hay expertos en un área determinada, las universidades no los han formado o los están formando mal; en otros países el problema son las cargas de trabajo excesivas que hacen imposible que los jueces tengan el tiempo para hacer un análisis concienzudo de estas pruebas; en algunos países el problema es cultural, más general, porque se da más importancia a la magia, a lo divino o a lo esotérico, antes que al conocimiento empírico fundado y/o hay una deficiente formación en la educación básica respecto al razonamiento con generalizaciones empíricas o científicas o fundadas; en otros países el problema es económico, porque no se ha invertido lo suficiente para tener buenos, variados y suficientes expertos y/o laboratorios de calidad. Y todos esos déficits pueden darse conjuntamente en cualquier combinación. Sin embargo, aunque no todos los problemas de la prueba pericial están relacionados con la calidad del conocimiento experto, todo sistema jurídico debería estar interesado en conocer la fiabilidad de los métodos, técnicas, teorías, etc., que son empleados por los peritos, en la completitud de la información con que se toma la decisión $y$, por supuesto, en que esta sea racional. 
El diseño normativo de las pruebas periciales, a propósito del razonamiento...

\section{Un presupuesto: la capacidad de los jueces}

Uno de los escenarios donde quizá más se ha debatido sobre la capacidad de los jueces para decidir sobre los hechos del caso es en EUA, donde en general el "juzgador de los hechos" es el jurado. ${ }^{3}$ Ahí precisamente ha surgido lo que se identifica como "paternalismo epistémico", es decir la política de tomar medidas para proteger a los jurados de sus malas decisiones. ${ }^{4}$ Esa protección sería, como en todo paternalismo, una protección de ellos mismos; ${ }^{5}$ pero, a diferencia de otros paternalismos relacionados con la acción, el objetivo sería evitar un mal razonamiento o una mala conclusión. Goldman, por ejemplo, delimita al paternalismo epistémico hacia los juzgadores de los hechos de la siguiente manera:

Si, en opinión de los legisladores, una cierta categoría de elementos
de juicio suele inducir al jurado a error, ellos están facultados para
exigir o permitir que tales pruebas se mantengan fuera del alcance
de los jurados. Este es un ejemplo de lo que llamo paternalismo
epistémico. [...] El tribunal sustituye con su propia expectativa
sobre el error del jurado la expectativa del jurado mismo, quienes
quizá hubiesen [de haber estado facultados para hacerlo] aceptado
información sobre el carácter del acusado, sus antecedentes penales
o su retractación de un reconocimiento de culpabilidad previo,
considerando que probablemente reduciría su probabilidad de
error (Goldman, 1991, traducción propia).

Es decir, bajo el presupuesto de que en determinadas condiciones el juzgador de los hechos cometerá errores, se limita su autonomía epistémica

3 Aunque la inmensa mayoría de los casos se deciden en el pre-trial y, por tanto, no hay un juicio oral (trial) seguido ante un jurado. Véase al respecto las estadísticas publicadas por el Bureau of Justice Statistics del U.S. Department of Justice, accesibles en https://www.bjs.gov.

4 Una parte de la doctrina considera incluso que las FRE dan perfecta cuenta de ello, véase Schauer (2006, p. 165), Mnookin (2006, pp. 134-145).

5 La idea de "protegerse de ellos mismos" es de Hart (1966). Aunque Hart se refería al paternalismo concerniente al razonamiento práctico, la idea es aplicable en términos generales al tipo de paternalismo relacionado con el razonamiento teórico, que es el que aquí nos interesa. 
con el fin de obtener resoluciones correctas sobre el caso en cuestión. En el ejemplo citado por Goldman, dicho límite es impuesto por el legislador y ejercitado por un juzgador profesional que debe "proteger" al jurado de sí mismo seleccionando la información que conocerá para decidir ${ }^{6}$. Por supuesto, hay otras posibles medidas que podrían tomarse, más allá de que el juzgador no tenga conocimiento alguno de la existencia de ciertas pruebas, como indicarle cómo tratar tales pruebas o su valor probatorio; sin embargo, el paternalismo epistémico ha optado por la exclusión de la información.

Una pregunta que surge de inmediato es dónde radicaría la incapacidad de los juzgadores de los hechos, i. e., de qué incapacidad se está hablando. Los factores relevantes a considerar parecerían ser cierto estado o condición de los sujetos que supongan límites o déficits cognitivos traducibles en una tendencia sistemática al error. Es decir, no se trata simplemente de errores ocasionales, ${ }^{7}$ sino de una cuestión general, sistemática y predecible. Por ello, deben diferenciarse esta tendencia generalizada a los errores de aquello que sobre juzgadores particulares enuncia Devis Echandía:

... el riesgo de la pereza física y mental de algunos juzgadores, que no hagan uso de la facultad legal para criticar [a la prueba pericial], sino que se faciliten el trabajo rindiéndole un exagerado servilismo. Pero este riesgo existe igual con otros medios de prueba [que] pueden ser aceptados servilmente o sometidos a rigurosa crítica, según la competencia y la preparación de cada juez. [...] [Es] por esta razón que no puede esgrimirse este peligro como

6 En el caso de un proceso judicial el objeto de protección último serían las partes en litigio y/o la sociedad en general. Aunque quizá baste aquí con defender que el interés de las medidas paternalistas epistémicas es acercarse lo más posible a la verdad (o la formación de creencias justificadas). Nótese que el ejercicio de las medidas paternalistas no siempre es posible, por ejemplo, cuando la admisibilidad de las pruebas se decide por el mismo sujeto que las valora.

7 Las causas de estos errores podrían ser sumamente variadas, como la mala memoria, la falta de atención, ciertas predisposiciones emocionales, la complejidad del caso o, incluso, la falta de habilidades de un juzgador concreto para cuestionar, etc. 
El diseño normativo de las pruebas periciales, a propósito del razonamiento...

algo exclusivo de la moderna prueba técnica o científica (Devis Echandía, 1972, p. 61).

Sea como sea, primero habría que saber qué capacidades cognitivas podrían considerarse relevantes a efectos de analizar si los actuales decisores son o no cognitivamente competentes para realizar la función que se les ha atribuido, es decir, valorar las pruebas periciales. Vale la pena subrayar que al juzgador no se le exige que produzca una prueba pericial, lo que se supone que tiene que valorar es la calidad de la inferencia hecha inductivamente a partir de la propia información que la conforma: los hechos relevantes sobre el caso, las generalizaciones independientes a los hechos del caso y las diversas relaciones entre ambas cuestiones. ${ }^{8}$ Esto es, sin duda alguna, considerablemente menos demandante epistemológicamente que lo primero, pues bastaría con determinar que las decisiones inferenciales del experto son (o no) razonables. $\mathrm{Si}$, en cambio, se duda de la capacidad de los jueces para realizar y/o evaluar inferencias, ello tendría consecuencias que irían mucho más allá de la prueba pericial, pues si aceptamos que todo el razonamiento probatorio es inferencial probablemente tendríamos que empezar a pensar en otro tipo de decisores o quizá en distintas estrategias normativas más contundentes sobre la institución probatoria. ${ }^{9}$

Una vez identificada la incapacidad relevante que se atribuye a los juzgadores de los hechos, la justificación del paternalismo epistémico exigiría disponer de datos empíricos sólidos que muestren la existencia de esa incapacidad, y no meras suposiciones al respecto. En el contexto anglosajón se han realizado varios estudios con el objetivo de comprobar o refutar que

8 En la misma línea, Dwyer (2008, p. 108). Para Dwyer, ello implicaría que el experto que realiza la inferencia correspondiente identifique los elementos probatorios relevantes, tome decisiones apropiadas sobre las inferencias que podrían ser hechas a partir de tales elementos y asigne probabilidades adecuadas a estas, además de establecer relaciones entre ellas.

9 En el contexto jurídico-procesal, la capacidad natural ordinaria del ser humano para hacer deducciones y/o inferencias probabilísticas en general se ha discutido para justificar la libre valoración de la prueba; por ejemplo, desde la psicología se han hecho investigaciones sobre patrones sistemáticos o tendencia sistemática de error cognitivo en el razonamiento ordinario, $v$. $g r$. la falacia de la afirmación del consecuente o la no consideración de probabilidades a priori. Al respecto, véase un brillante artículo de Cohen sobre la libertad en la valoración de las pruebas y su legitimidad (Cohen, 1983). 
los jurados como juzgadores de los hechos son fácilmente impresionables cuando se les presenta una prueba etiquetada como "científica". Ahora bien, nótese que la incapacidad que se pretende determinar aquí no es relativa al control de las inferencias realizadas por los expertos, sino una sobrevaloración epistémica sobre un tipo de pruebas periciales que podría tener múltiples causas. De cualquier manera, los resultados de dichos estudios no son de ninguna manera concluyentes (algunos confirman la hipótesis y otros la refutan), ${ }^{10}$ lo que no debería ser muy sorprendente si tomamos en cuenta que el jurado es solo una muestra de la sociedad. ${ }^{11}$

Todo lo anterior no desconoce de ninguna manera que los usos que se han hecho del conocimiento experto en el proceso judicial han conducido a errores en el sistema, pero por causas diversas a la supuesta incapacidad de los jueces de los hechos de controlar las inferencias. Entre ellas se encuentra: que el sistema judicial ha empleado sistemáticamente información de fiabilidad baja o desconocida; que los peritos hacen afirmaciones que no cuentan con apoyo empírico en su área; que los peritos sistemáticamente asumen comportamientos que buscan favorecer a toda costa a su parte; que los peritos sufren de ciertos sesgos cognitivos dada su contaminación con determinada información del caso o que las pruebas periciales tardan mucho en ser realizadas (Duce, 2018); que los litigantes no tienen la formación y/o las destrezas adecuadas para cuestionar a los expertos, o incluso que desconocen el caso que están litigando, etc. Sin embargo, ninguno de esos genuinos problemas apunta directamente a que los jueces son incapaces cognitivamente de realizar su función; apuntan, más bien, a un conjunto de malas prácticas de los diversos actores y quizá a una mala formación de los juristas en general en el ámbito probatorio.

${ }^{10}$ Aparece un listado sumamente completo e interesante de los distintos tipos de estudios realizados en Estados Unidos (Schauer y Spellman, 2013).

${ }^{11}$ Un ejemplo realmente llamativo sobre el jurado como muestra de la sociedad es Australia. En un reciente estudio empírico sobre las percepciones de los jurados sobre las pruebas periciales se da cuenta de que el 45,5\% de los jurados encuestados habían estudiado ciencias o matemáticas en educación superior y un 4,8\% de ellos eran empleados profesionales en un área científica. Mientras que el $74,1 \%$ de los jurados tenía conocimientos en ciencias o matemáticas gracias al énfasis en dichas áreas en la escuela australiana. Véase Freckelton, Goodman-Delahunty, Horan y McKimmie (2016, p. 14). 
El diseño normativo de las pruebas periciales, a propósito del razonamiento...

Es más, si los jurados no fuesen capaces de valorar las pruebas periciales todavía podría decirse que la tarea debe ser realizada por jueces profesionales, pero cuando ya la tarea es realizada por un juez profesional y desconfiamos sistemáticamente de este, ¿quién entonces debería realizar la función?, ¿otro experto? Más allá de los problemas prácticos que supondría crear un sistema donde todos los jueces fueran expertos en la materia pericial que deben juzgar y que, para ello, deberíamos tener juzgadores ad hoc, finalmente todo tipo de jueces, como todo ser humano, es susceptible de sufrir sesgos cognitivos en diferentes situaciones donde hay mucha información que se tiene que procesar, cuando se necesita actuar de manera rápida, cuando se tiene que recordar información disponible anteriormente o incluso cuando se llenan lagunas en ausencia de determinada información. ${ }^{12}$ Todas esas situaciones están claramente presentes en los procesos judiciales, con independencia del tipo de jueces que se tienen.

Si desconfiamos sistemáticamente de la capacidad de nuestros jueces, entonces, debemos buscar otras maneras para el funcionamiento adecuado de los sistemas y apartar definitivamente a los incapaces de realizar la función en juego; esto supondría, obviamente, repensar toda la estructura del proceso judicial para saber quién tendría que decidir los casos jurídicos que lleguen hasta los tribunales. Lo que no tiene ningún sentido es desconfiar sistemáticamente de la capacidad de los jueces legos (i. e. no expertos en el campo de conocimiento del perito) y mantenerlos en la toma de decisiones judiciales. Sin embargo, dado el giro copernicano que esto supondría, los fundamentos que debemos considerar para llevarlo a cabo no deben ser netamente políticos, tiene que haber un sustento empírico que muestre dicha incapacidad, más allá de perezas mentales, incentivos institucionales, corruptelas, etc., de algún juez o conjunto de jueces.

Si adoptamos un sistema de confianza en las capacidades de los jueces para tomar decisiones también con conocimiento experto, entonces, me parece muy urgente dejar la "estrategia victimista" consistente en repetir el mantra "los legos vs. los expertos", a la vez que de muy diversas maneras justificamos que los primeros realicen pocos o nulos esfuerzos por com-

${ }^{12}$ Sobre los sesgos cognitivos en el razonamiento judicial puede verse Stafford, Jules y Robin (2018). 
prender a los segundos. Y, por el contrario, creo que debemos repensar nuestro diseño institucional teniendo muy claros los objetivos que se buscan y los diversos problemas que siempre enfrentaremos, con la prueba pericial y sin ella, como la falibilidad de cualquier sistema por más perfecto que teóricamente pudiese ser y los sesgos cognitivos de los diversos sujetos que participan en el funcionamiento del mismo.

Estas asunciones tienen que ir precedidas por una delimitación previa referente a las pruebas periciales que, aunque quizá es obvia, no siempre se toma en cuenta: es falso que nuestros jueces lidien con el mundo de la expertise. En otras palabras, no todo el conocimiento experto que se ha desarrollado en la actualidad tiene cabida en el proceso judicial, solo una pequeña parte del mismo; no todo el conocimiento experto que tiene un experto es relevante para el caso concreto, solo una parte del mismo; no todo el conocimiento del caso concreto es experto, sino que generalmente, además de este, hay disponible otro cúmulo de conocimiento no-experto relevante. Y normalmente el tipo de conocimiento experto que entra al proceso ha sido ya tratado previamente por nuestros jueces al ser repeat players y tener como función decidir constantemente casos a partir de él. Esto último no convierte en expertos a nuestros jueces, pero sí les debería permitir una mayor sofisticación en el tratamiento de dicho conocimiento.

Es muy posible que los sistemas actuales no estén logrando la sofisticación que se requeriría para tratar las pruebas periciales, pero quizá ello no se deba a la incapacidad de los jueces, sino a una suma de errores, incentivos perversos y desconocimiento sobre el funcionamiento del conocimiento experto que ha configurado el diseño y las prácticas procesales actuales. Y, desde luego, una forma de incidir en todo ello es cambiando el sistema jurídico que regula al menos determinados aspectos de la prueba pericial, lo que, si además fuera acompañado de una estrategia formativa fuerte, ${ }^{13}$ podría darnos mejores resultados en el tratamiento jurídico-pro-

${ }^{13}$ Me refiero a la educación que deberían recibir jueces, abogados y fiscales sobre el conocimiento experto fuera de los procesos judiciales particulares, en su formación continua ofrecida por las escuelas judiciales, de fiscalía o los colegios profesionales. Es sumamente llamativo, por ejemplo, que estando tan continuamente presente la prueba de ADN en los procesos judiciales, los operadores jurídicos no hayan tenido nunca algún curso que les informe mínimamente sobre la materia. 
El diseño normativo de las pruebas periciales, a propósito del razonamiento...

cesal del conocimiento experto. La formación de los jueces en materia probatoria es una necesidad urgente, pero no es este el lugar para profundizar en ello; mi objetivo es más modesto: hacer algunas sugerencias sobre el diseño normativo actual.

\section{Algunas herramientas procesales para ayudar a la comprensión judicial}

La prueba pericial como fuente de conocimiento es un testimonio, es decir, obtenemos la información relevante a partir de un tercero. ${ }^{14} \mathrm{Y}$ aquí cobra importancia tomar en consideración una de las más claras enseñanzas que nos ha dado la llamada epistemología del testimonio, ampliamente desarrollada en los últimos años: las cualidades o insumos epistémicos que ese tercero tiene sobre lo que afirma no son heredables para la audiencia que lo escucha. Es decir, las razones que ese tercero tiene para creer que P no son simplemente heredadas por el sujeto que escucha a ese tercero afirmar que P. Si esto fuese así, el núcleo de la epistemología del testimonio radicaría de forma exclusiva en el hablante (concretamente, en la justificación de su estado mental), pudiendo la audiencia heredar la justificación de las afirmaciones realizadas por este. Sin embargo, hay varios argumentos en contra de dicha tesis:

1. Pese a que haya un conjunto de pruebas sobre determinada afirmación, un sujeto puede tener creencias sobre esa afirmación contrarias a las pruebas existentes y, a la vez, fundar las afirmaciones que realiza en esas pruebas disponibles y no en sus propias creencias. Entonces, pese a que un hablante no tuviera una creencia justificada sobre $\mathrm{P}$, la audiencia podría estar justificada en creer que $\mathrm{P}$ a partir únicamente de lo que dice el testimonio respectivo.

2. Aun cuando el hablante tenga creencias justificadas sobre una afirmación, es posible que por diversas cuestiones sea la propia audiencia quien impida la transmisión de dicha justificación y, por

14 Para profundizar en esta cuestión, puede verse Vázquez (2015, pp. 44 y ss., 149 y ss. y 211 y ss.). 
ello, pese a la justificación del hablante, la creencia de la audiencia no esté justificada.

3. La creencia transmitida no implica de ninguna manera la transmisión a la audiencia de posibles pruebas o razones que tuviera el propio hablante para derrotar dicha creencia o, al menos, cuestionarla. ${ }^{15}$

Dados estos serios problemas de la llamada "concepción hereditaria" del testimonio, hay que buscar dar cuenta del conocimiento testimonial considerando a los dos agentes epistémicos en juego, es decir, el juez como audiencia y el perito como hablante. ${ }^{16}$ En el contexto procesal todo ello se traduciría en que el juez debe tener sus propias razones suficientes para creer justificadamente las afirmaciones o conclusiones periciales; y para ello, la práctica de las pruebas en contradicción es quizá el mejor momento procesal para obtener adecuadamente el tipo de información que requiere. Vamos por partes.

Como se ha dicho antes, la tarea judicial con la prueba pericial consistiría básicamente en controlar el razonamiento inferencial realizado por los expertos, lo que supone controlar también las premisas en juego. Aunque quizá sea simplificar demasiado el razonamiento pericial, una manera que podría resultar asequible para los juristas sea distinguir entre la premisa mayor del razonamiento compuesta por generalizaciones independientes a los hechos del caso y una premisa menor compuesta por los hechos del caso, ambas llevarían a una conclusión. Un sencillo ejemplo podría ser "cuando mentimos hay un esfuerzo consciente que se refleja en un aumento de la

${ }^{15}$ Estos problemas son ampliamente analizados por Lackey (2008).

${ }^{16}$ No se trata, como decía Stein en su clásica obra El conocimiento privado del juez (1990, p. 62), de que el juez convierta en percepción propia la explicación técnica del perito. Es cierto que el juez, como todo agente, para conocer o entrar en contacto con lo que otros le dicen, con lo que le es comunicado lingüísticamente, depende operativamente de la percepción; pero ¿qué importancia epistémica tendría ver un informe pericial o escuchar lo que dice el perito?, ¿habría que reducir el estatus epistémico de las creencias, la justificación o el conocimiento obtenido mediante el testimonio de un experto a la percepción del juez? Por supuesto, el juez requeriría de la inmediación para conocer del testimonio, oral o escrito, que le es presentado, pero la justificación de la información que mediante este adquiriera es una cuestión distinta. 
El diseño normativo de las pruebas periciales, a propósito del razonamiento...

presión sanguínea", que era la generalización en juego en el famoso caso Frye, donde se pretendía presentar como prueba pericial un entonces novedoso detector de mentiras. ${ }^{17}$ Por supuesto, muchas veces la premisa mayor será mucho más compleja que una única afirmación, será un conjunto de afirmaciones cada una de las cuales podría integrar una inferencia inductiva independiente. ${ }^{18}$ De cualquier manera, nótese que antes de valorar cómo se ha aplicado al caso concreto, hay que tener los datos relevantes sobre la justificación de la generalización en juego; podríamos decir que esto es condición necesaria pero no suficiente de una valoración judicial sobre las pruebas periciales, todavía faltaría por ver si en el caso concreto, por ejemplo, la medición del aumento de la presión sanguínea de Diego permite inferir que él ha mentido. Sin embargo, cuando la premisa mayor es cuestionada y mostrada inválida, entonces ya no haría falta el análisis de la premisa menor, como en el caso de los detectores de mentiras ejemplificado.

Si somos conscientes de la información que necesitamos, ya podríamos exigir a las partes cómo deben conformar el ofrecimiento de sus pruebas periciales y/o sus informes periciales: explicitando claramente la inferencia o las inferencias en juego. Sin embargo, a efectos de subsanar cualquier déficit de información y para comprender adecuadamente el razonamiento pericial, la práctica de las pruebas periciales será fundamental. La oralidad

${ }^{17}$ El caso Frye (1923) fue el primer caso en la experiencia estadounidense donde se estableció jurisprudencialmente un criterio de admisibilidad de las pruebas periciales atendiendo a la calidad de las mismas: la aceptación general de la comunidad experta relevante. Un significativo salto que ha llevado a centrar la atención en las comunidades expertas más allá de los peritos particulares. Al respecto, puede verse Vázquez (2015, pp. 92 y ss.).

${ }^{18}$ Considérese como ejemplo el siguiente listado en casos en los que se debe determinar la edad biológica de una persona sin documentación oficial al respecto: 1. La determinación de la edad de un joven sin ningún registro oficial al respecto solo puede llevarse a cabo mediante estimaciones sobre su edad biológica a partir del grado de maduración de ciertas estructuras anatómicas. 2. Existe consenso en la necesidad de utilizar varias técnicas de diagnóstico, cada una con sus propios medios auxiliares, y en combinar el resultado de todas ellas para sustentar un diagnóstico fiable. 3. Uno de esos métodos es la valoración Atlas de maduración ósea de Greulich y Pyle, consistente en comparar la radiografía del individuo con las radiografías estándar para cada edad y comprobar con qué edad "tipo" coincide. 4. Otro de esos métodos es la radiografía de mano izquierda mediante el método Greulich-Pyle TW2-RUS, basada en el estudio del grado de maduración ósea de los huesos del carpo, etc. Véase el ejemplo completo Vázquez (2015, pp. 190 y ss.). 
en combinación con la contradicción puede colaborar a que se incorpore adecuadamente información relevante sobre el razonamiento pericial. Pese a que es el principio de oralidad el que más difusión ha tenido, este no tiene, en sí mismo, efectos epistemológicos, pues puede ser regulado y aplicado de manera tal que no se aprovechen las oportunidades que potencialmente ofrece para una mejor práctica de las pruebas; por ejemplo, obligando a formular y seguir una lista cerrada de preguntas, sin posibilidad de repreguntar o limitando la contradicción exclusivamente para las partes al concebir al juzgador en una actitud totalmente pasiva. Por ello, es fundamental enfatizar la combinación oralidad- contradicción como una estupenda dupla que permitiría tanto a los jueces como a los fiscales y abogados un mejor acercamiento al contenido de las pruebas periciales. ${ }^{19}$

El principio de contradictorio es, evidentemente, una de las más importantes garantías procesales de las partes al permitir que cuestionen las pruebas presentadas por su contraparte y también que puedan de alguna manera controlar el razonamiento judicial en aquellos casos en que el juez lo refleja a través de las preguntas que hace a las propias partes, los testigos y los peritos. Por eso el ejercicio del principio de contradictorio también debe servir como herramienta cognoscitiva a los jueces, es decir, les debe permitir subsanar sus déficits de información sobre las pruebas periciales admitidas. Desafortunadamente, no siempre pueden hacer tal subsanación únicamente a través de las partes, por ejemplo, porque no cuestionan adecuadamente y el juez sigue teniendo dudas o incluso cuando pese al cuestionamiento de las partes las dudas persisten. Si asumimos que los jueces deben comprender las pruebas presentadas para tomar una decisión racional, entonces lo mejor que puede pasar a las partes es que los jueces

${ }^{19}$ Como se verá, asumo aquí una visión epistémica del contradictorio, incompatible con las concepciones que lo ven como espacio de combate entre las partes. En la misma línea, por ejemplo, Ferrer sostiene que: "[d]ado que la finalidad institucional principal de la fase de prueba en el proceso judicial es la averiguación de la verdad, el sistema procesal jurídico, como no podía ser de otra manera, importa en forma de instituciones jurídicas los mecanismos epistemológicos necesarios para alcanzar esa finalidad. En este caso, puede decirse que el modo de implementar jurídicamente mecanismos que faciliten la corroboración es el denominado principio de contradicción" (Ferrer, 2007, p. 86). Sobre este tema pueden consultarse un par de trabajos de Tonini $(2003 ; 2011)$. 
El diseño normativo de las pruebas periciales, a propósito del razonamiento...

resuelvan sus dudas durante la práctica de las pruebas y no que, habiéndose quedado con ellas, las satisfagan preguntando a un experto de su confianza o incluso consultando en otras fuentes que, evidentemente, las partes desconocerán y no tendrán ninguna posibilidad de contradecir. Por ello, no podemos aspirar siquiera a que los jueces permanezcan totalmente pasivos durante la práctica de la prueba pericial.

Dicho lo anterior, podría haber casos complejos en los que el desconocimiento del juez impidiese siquiera generar dudas relevantes para la adecuada comprensión de las afirmaciones periciales. En esos casos, sería muy oportuno que el sistema jurídico previese la posibilidad de contar con un "consultor experto" que pudiera educar básica y rápidamente al juez sobre las cuestiones más elementales de un área de conocimiento. Es decir, no se trataría de un perito sino de un experto con una función muy concreta y distinta a la de ofrecer pruebas sobre los hechos concretos del caso: la función de dar al juez las nociones más básicas de un área o aspectos relevantes de la misma para que posteriormente sea capaz de comprender adecuadamente lo que los peritos le dirán e incluso preguntar razonablemente. ${ }^{20}$ Un experto así no debería conocer los hechos del caso concreto, evitando con ello cualquier contaminación del conocimiento que pondrá en manos del juez. Por supuesto, habría que discutir cómo sería nombrado y garantizar en todo momento la participación de las partes, no necesariamente contradiciendo sus afirmaciones, pero sí en su nombramiento y en todo contacto que tuviese con el juez respectivo, quien debería comunicar siempre a las partes cualquier reunión con el consultor.

Por otro lado, también podría haber casos en los que el ejercicio del contradictorio trajese más dudas cuya adecuada resolución requiriese más operaciones periciales y, para esas situaciones, podría preverse la posibilidad de realizar lo que podría identificarse como "meta-periciales". Es

${ }^{20}$ Esta figura existe en la práctica procesal estadounidense, aun cuando no está prevista específicamente en la normativa, se considera que las facultades de los jueces para conducir las cuestiones preliminares de los casos en conjunto con la facultad de nombrar expertos les permite nombrar a los llamados "technical advisors". Ahora bien, es una figura que tiene lugar en la etapa de admisión de las pruebas y no en la práctica de las mismas, lo que es relevante puesto que quien la usa no es el juzgador de los hechos, sino el juez que tiene que decidir la admisibilidad de las pruebas. 
decir, que haya una nueva prueba cuyo objeto exclusivo de análisis sea dar respuesta a esas preguntas concretas a partir de las pruebas periciales inicialmente admitidas y practicadas. Vale la pena enfatizar que esta opción debería estar disponible únicamente para aquellos casos en que el contradictorio entre las partes genere más dudas sobre las afirmaciones realizadas por los expertos; no se trataría entonces de una tercera prueba pericial que inicia desde cero, sino una revisión sobre lo realizado por los dos expertos u ampliación de lo mismo.

Algunos sistemas actuales tienen al llamado "perito tercero en discordia" que se utiliza cuando el juez tiene alguna duda después de escuchar a los peritos que han participado o cuando hay un desacuerdo entre ellos. Sin embargo, en mi opinión, ese sistema ha generado incentivos perversos para los jueces, quienes a veces dan mayor valor probatorio al tercero en discordia simplemente porque es un perito oficial y, otras ocasiones, resuelven de manera netamente numérica asumiendo como correcto aquello que afirma la mayoría. En este tipo de prácticas podría subyacer la idea de que todo desacuerdo entre los peritos es necesariamente debido a una parcialidad de alguno de ellos, lo que es una concepción sumamente reductivista de los desacuerdos entre los expertos.

Los desacuerdos entre los expertos no son una anomalía en el funcionamiento de la ciencia, al contrario, la historia del progreso del conocimiento está repleta de ellos, son parte integral de la misma. ${ }^{21}$ Son explicables no solo por la existencia de diversas escuelas de pensamiento, sino porque los datos son susceptibles de interpretaciones alternativas o porque todavía no hay un consenso sobre la suficiencia de la evidencia con la que cuentan o porque hay diferentes métodos que pueden ser aplicables para logar un mismo objetivo o porque se pueden inferir diversas conclusiones a partir de ciertos datos. Ahora bien, también puede haber desacuerdos meramente aparentes porque, por ejemplo, alguno de los expertos ha utilizado información diferente a la utilizada por el otro perito (simplemente porque le fue dada esa y no otra). Por ello, uno de los primeros objetivos a lograr en el análisis de los desacuerdos entre peritos sería identificar si se está frente a un genuino desacuerdo o ante un desacuerdo aparente. En la práctica 
El diseño normativo de las pruebas periciales, a propósito del razonamiento...

esto no se hace, el derecho sistemáticamente ha tratado de huir de los desacuerdos ofreciendo a los jueces distintas salidas para ello, como el perito tercero en discordia o el "perito único", sin darse cuenta que no hay manera de huir: al hacerlo se está optando por uno de los lados disponibles, sin saberlo y, por supuesto, sin hacerlo de manera justificada.

$\mathrm{Si}$ asumimos que los desacuerdos entre expertos no solo pueden ser normales sino incluso sanos, dado que nos muestran un panorama más amplio sobre lo que podemos saber respecto de una misma cuestión, habría que buscar maneras de gestionarlos jurídicamente de mejor manera. Y, en mi opinión, una forma de hacerlo es que siempre que se dé tal situación se lleve a cabo una junta para propiciar un escenario de diálogo entre ellos y con ellos. Esta estrategia no es nada novedosa, desde 1980 en Australia surgió el llamado "hot-tub" que, básicamente, es la reunión del juez y las partes con los peritos con el objetivo claro de delimitar y/o aclarar los desacuerdos entre ellos (Hazel, 2013). La idea de base sería algo así como replicar un escenario que debería ser familiar para los expertos: un sano debate de las ideas y/o de la información considerada en sus afirmaciones. Ahora bien, puesto que el proceso judicial no es y no puede ser de ninguna manera un contexto académico, hay que buscar la manera más efectiva para que dicho debate sea informativo para el juez que debe decir. A esos efectos se le debería pedir a los peritos que desacuerdan que antes de la reunión presenten al juez un informe conjunto sobre sus puntos de acuerdo y de desacuerdo y una suerte de justificación de los mismos, ${ }^{22}$ de manera que el juez participaría en la reunión con los expertos teniendo la información relevante para dirigir el debate y no viéndose sistemáticamente sobrepasado por los expertos.

Hay distintas maneras de llevar a cabo la junta de peritos: 1) permitir que se pregunten entre ellos; y/o 2) hacer preguntas específicas para ellos; y/o 3) debatir sobre los informes periciales. En el sistema inglés, donde ha estado en práctica desde hace unos años, todas las posibilidades están abiertas para el juez, quien tiene dentro de sus facultades de case managment elegir la que cree que será la mejor dinámica para afrontar el

22 Esta disposición está actualmente vigente en Inglaterra, véase el artículo 35.12 (3) de las Civil Procedure Rules y, sobre todo, los numerales 9.1 a 9.8 de la Practice Direction 35. 
desacuerdo en ese caso. ${ }^{23}$ Los datos que arrojan los estudios que se han realizado sobre la experiencia de jueces y abogados es sumamente positiva, el $83 \%$ de los miembros de la judicatura encuestados respondieron que el hot-tubbing mejoraba la calidad de las pruebas periciales, mientras que el $84 \%$ de los abogados respondió de la misma manera. ${ }^{24}$ Vale la pena enfatizar que esta técnica se ha mostrado útil para afrontar diferencias sustantivas entre los expertos, pero no para cuestiones relacionadas con su falta de credenciales o experiencia o su falta de independencia o la afectación a algún tipo de sesgos.

La evidencia empírica sobre el funcionamiento del hot-tubbing en Inglaterra es claramente persuasiva, pero obviamente no podemos inferir a partir de ella que también funcionará en otros sistemas. En mi opinión, no obstante, debemos probar si funciona en nuestros contextos, dado que constituye un mucho mejor mecanismo para tratar los desacuerdos entre expertos, para lograr una genuina justificación sobre las inferencias que hacemos a partir de ellos. Como cualquier otra regla procesal, una vez implementada deberíamos medir empíricamente su funcionamiento real para conocer sus éxitos, pero también sus debilidades o fracasos y, en su caso, modificarla.

Finalmente, hay algunos tipos de casos en los que la complejidad técnica es tal que, incluso poniendo en práctica todas las herramientas antes mencionadas, se presentan dificultades serias de comprensión para un juez lego. En esos casos queda aún una opción: que un experto sea incorporado como miembro del tribunal. Por supuesto, surgen aquí varias cuestiones: ¿cómo y cuándo se nombrará a un experto que potencialmente hará de juez?, ¿cómo se garantizará la participación de las partes?, ¿su decisión será obligatoria para los jueces legos? Todas son preguntas muy relevantes en una decisión que debería ser muy excepcional y estar bien fundada: la incorporación al tribunal de un experto que, claramente, asumiría las responsabilidades de participar junto con un juez (o conjunto de jueces) en la

${ }^{23}$ Sobre el "case management" pueden verse van Rhee (2007), Denyer (2012).

${ }^{24}$ Véase "Concurrent Expert Evidence and 'Hot-Tubbing' in English Litigation since the 'Jackson Reforms'. A Legal And Empirical Study”, desarrollado por el Civil Justice Council, 25 de julio de 2016, pp. 58 y 59. 
El diseño normativo de las pruebas periciales, a propósito del razonamiento...

toma de una decisión judicial. Tampoco esta figura es un invento personal: está prevista en el ordenamiento danés, son llamados assessors, expertos que son pagados por el gobierno en casos muy complejos y que de ninguna manera suplen a los peritos de parte (Movin Østergaar, 2016, pp. 319-341).

A diferencia de los consultores mencionados párrafos arriba, los asesores daneses asisten al juicio oral para escuchar las pruebas y luego participan en las deliberaciones judiciales, con el aspecto positivo de que si los “jueces tienen dudas sobre aspectos técnicos del caso, no solo pueden preguntar al experto, sino que también tienen la oportunidad de participar de un genuino debate con los asesores sin preocuparse de revelar su opinión sobre la decisión del caso" (Movin Østergaar, 2016, p. 331, la traducción es mía). Además de esto, los argumentos que se ofrecen para justificar dicha figura en el ordenamiento jurídico son la legitimidad de una decisión en la que explícitamente asumen la responsabilidad el (o los) experto(s) en conjunto con los jueces y el consenso entre los representantes del mundo jurídico y del mundo científico-tecnológico. Según Movin Østergaard, la experiencia danesa está conformada sistemáticamente por decisiones de consenso entre los jueces profesionales y los asesores, sin embargo, cuando se ha dado un disenso y a efectos de la segunda instancia, debe quedar claro quién está en desacuerdo en la decisión tomada (Movin Østergaar, 2016, p. 332).

El nombramiento de un asesor a la danesa tendrá unos u otros problemas en atención al sistema jurídico en el que buscase implementarse: por ejemplo, no es lo mismo su implementación en un diseño donde se prevé que un solo juez tome la decisión sobre los hechos que en un diseño donde el juzgador de los hechos es colegiado. En un sistema en donde la decisión sea tomada por dos personas, el juez profesional y el asesor incorporado al tribunal, debería primar la decisión del juez lego quien, por obvias razones, deberá ofrecer una motivación reforzada de su decisión con la claridad suficiente en la explicitación de sus razones que permita luego a la segunda instancia realizar una revisión adecuada. Sin dudas, es una figura controversial, pero estaría dotada de mayor legitimación (y racionalidad) que la deferencia a alguien que se tiene como experto y que el mismo sistema prevé que no es quien debe decidir.

Todas estas figuras van más allá de la tradicional opción entre peritos de parte y peritos oficiales, previendo distintos roles para distintas necesidades 
de la decisión judicial, buscando a la vez incentivar una genuina sana crítica hacia los expertos a la vez que nos tomamos en serio su conocimiento y participación. Evidentemente, hay que tener en cuenta que no en todos los casos están en juego todas las piezas de este escenario; para la activación de las distintas figuras habría que tomar en cuenta algunos factores como la complejidad del conocimiento experto en juego, el carácter determinante de la prueba pericial para el caso, el monto del litigio, el tiempo disponible, el grado en el que haya una significativa diferencia de opiniones entre los peritos, etc. De cualquier manera, un sistema jurídico que prevea las diferentes opciones aquí mencionadas está ofreciendo a los jueces herramientas necesarias para afrontar diversos grados de complejidad que pueden presentarse en las pruebas periciales y, quizá más importante aún, indicando explícitamente que el objeto de valoración de las pruebas periciales son las afirmaciones realizadas por los expertos.

\section{Sobre la información que funda una prueba pericial}

Como se mencionó anteriormente, las diversas herramientas presentadas en el epígrafe anterior pueden ser complejas, costosas económicamente e implicar también costos de tiempo para el proceso. Por ello, deben ser empleadas con prudencia y solo cuando la complejidad técnica del caso lo aconseje. Antes de llegar a la utilización de esas herramientas, sin embargo, conviene que de forma general nos ocupemos de las pruebas periciales desde el primer momento: qué se pregunta a los expertos, qué información sobre el caso les brindamos para sus operaciones periciales y cuáles son las obligaciones que les imponemos en toda su actividad. Veamos cada una de estas cuestiones.

En una reciente encuesta a expertos que han participado como peritos en los tribunales estadounidenses, cuando se les preguntó sobre las razones por las cuales habían rechazado participar como peritos en un proceso judicial determinado, el $49 \%$ respondió que habían rehusado dado que "la cuestión estaba fuera de mi área de expertise" (Seidman y Lempert, 2018, p. 45). Una respuesta muy preocupante, puesto que toda la actividad 
El diseño normativo de las pruebas periciales, a propósito del razonamiento...

pericial dependerá de este primer acercamiento entre los expertos y los juristas. Aunque en el mundo ideal ni siquiera debería mencionarse, es obvio que los abogados deben conocer amplia y profundamente el caso que defenderán: solo de esta manera serán capaces de identificar las lagunas probatorias que deben suplir y evaluar a qué tipo de expertos deben recurrir. Seidman y Lempert concluyen, a partir del dato arrojado por su encuesta, que la frecuencia de la respuesta sugiere que "un sistema que ayuda a los abogados y a los jueces a identificar a reconocidos expertos con conocimiento específicamente relevante para el caso incrementaría la eficiencia en la búsqueda de asesoramiento y podría promover la mejora de las pruebas periciales en el proceso judicial" (Seidman y Lempert, 2018, p. 45, la traducción es mía); lo que no resulta nada claro es cómo sería el sistema que sugieren, ¿están pensando en un mero listado de expertos disponibles para peritar, que ya existe en la mayoría de nuestros sistemas? o ¿la propuesta es más sofisticada y piensan en algún ente que ayude a identificar el experto adecuado para el caso específico?

Si tomamos en cuenta la experiencia que se ha tenido en muchos de nuestros países con el nombramiento del perito oficial y la dificultad de gestionar los listados, parecería poco aconsejable optar por esa estrategia para la selección de cualquier experto. ${ }^{25}$ En mi opinión, no habría que priorizar el ofrecimiento de esos mecanismos a las partes sino establecer un diseño institucional que favorezca la tarea de los abogados, por ejemplo, para determinar claramente cuál será el objeto del peritaje y para disponer de la información relevante para la realización de la prueba pericial, lo que se conseguirá si mejoramos en general las actividades que llevan a la conformación del conjunto de pruebas.

Si el juicio oral consistirá en una audiencia concentrada, entonces el caso debe llegar a ella lo más claro y pulido posible; y, a esos efectos, los actos procesales anteriores a aquella son determinantes para la conformación del conjunto de elementos de juicio que dará forma a las cuestiones de hecho y de derecho. Siempre podría suceder que en dicha dinámica las partes lleguen a un acuerdo o se determine que no hay un caso litigioso,

25 Sobre las dificultades de un sistema de nombramiento de expertos por los jueces, véase Vázquez (en prensa). 
pero para ello hay que tener toda la información relevante disponible que permita tomar las decisiones de la manera más racional posible. En este sentido, disponer de un conjunto de información completa debe ser la aspiración de cualquier diseño institucional serio: no se trata solo de tomar decisiones con cualquier conjunto de información, sino que la decisión se adopte con toda la información relevante disponible. ${ }^{26}$

Tradicionalmente en nuestros sistemas esa preocupación ha sido de alguna manera puesta en manos del juez, a través del ejercicio de algunos de sus poderes probatorios; sin embargo, en los últimos años las reformas procesales que hemos vivido han disminuido sistemáticamente el poder del juez de ordenar la incorporación de pruebas no solicitadas por las partes o incluso de sugerir la incorporación de pruebas que complementarían el conjunto presentado por las partes y, en cambio, han aumentado el control de las partes sobre la presentación de pruebas. En ese escenario, si al desiderátum epistemológico de tomar decisiones con un conjunto de pruebas completo sumamos una preferencia por evitar a toda costa que las partes terminen ganando un caso simplemente por utilizar argucias litigiosas, como sacar información nueva de forma sorpresiva durante el juicio oral o esconder información relevante, hay que establecer medidas para que las partes conozcan lo antes posible cuáles son las pruebas disponibles que fundan o refutan sus pretensiones, que tengan acceso a ellas y, por supuesto, que se cierre lo antes posible el conjunto probatorio que estará en juego durante todo el proceso judicial.

En los sistemas anglosajones, en los que tradicionalmente las partes han controlado las pruebas que presentan en un juicio, se ha impulsado mediante la institución del discovery o disclosure (que ha sido traducida como "descubrimiento probatorio") los objetivos de lograr la completitud del conjunto de pruebas y de evitar el "risk of surprise at the trial". Como su nombre sugiere, tiene como objetivo que las partes se descubran mutuamente la información que tienen durante el llamado pre-trial. Según

${ }^{26}$ Ello supone que el objetivo de la institución probatoria es la averiguación de la verdad y que el proceso judicial no es meramente un instrumento para resolver disputas personales entre las partes. Si el objetivo fuera únicamente ese, por supuesto, no necesitaríamos un diseño institucional refinado, dado que no importaría la corrección sustantiva de la decisión, sino la conformidad de las partes sobre la decisión. 
El diseño normativo de las pruebas periciales, a propósito del razonamiento...

Glacer, los partidarios del discovery estadounidense le atribuyen diversos beneficios:

Desde el punto de vista de los litigantes, permitiría traer información y pruebas no conocidas de otra manera, reduciendo el factor sorpresa en el juicio. Desde el punto de vista de la administración de justicia, el descubrimiento probatorio aliviaría las cargas de casos de los tribunales incrementando los acuerdos y reduciendo las apelaciones de veredictos, todo ello con el aumento en la calidad de los acuerdos y de los juicios... (Glaser, 1968, p. 83, la traducción es mía).

No es este el espacio para ahondar en esta figura procesal. Baste decir que con un mecanismo de este tipo las partes pueden tener un panorama amplio para evaluar de la mejor manera si necesitan una prueba pericial y qué información darán al experto, sin ir a ciegas o con información incompleta derivada únicamente de la poca información que en la práctica se suele dar en la demanda o en su contestación, cuando de un caso civil se trata, o por no haber tenido acceso a la llamada unused information obtenida en la investigación policial, cuando estamos ante un caso penal. ${ }^{27}$ Por supuesto, no tener toda la información relevante disponible en el momento en que las partes deciden si presentan o no una prueba pericial puede conllevar que no se elija bien la expertise pertinente dado que lo que será el objeto del peritaje es incierto o parcial y, por supuesto, que el contenido

${ }^{27}$ La "unused information" es la información obtenida mediante las investigaciones policiales que la propia policía o el fiscal encargado de la investigación desecha por considerar irrelevante para la presentación de su caso pero que, sin embargo, la parte investigada podría considerar relevante y a la que debe tener acceso, de cualquier manera, para juzgar su relevancia.

En algunos sistemas en los que los fiscales tienen el deber de actuar imparcialmente, se podría argumentar que no hace falta asegurar el acceso a la unused information porque debería ser información genuinamente irrelevante. Sin embargo, la carga excesiva de trabajo de los fiscales o incluso un mero descuido humano podrían llevar a descartar como irrelevante información que es genuinamente relevante y, por ello, aún en esos sistemas es importante el acceso de las partes a esa información. 
de un peritaje sea fragmentario como resultado simplemente de que la información que el experto tuvo a disposición fue también incompleta. ${ }^{28}$

Ahora bien, no solamente hay que empoderar a las partes en lo relativo al acceso a la información para que planteen su caso de la mejor manera, sino que también se debería establecer una relación mucho más fluida con el juez a efectos de determinar el objeto del peritaje. Es decir, se podría pensar en un modelo y/o práctica en el que haya cierto control judicial genuino sobre qué información del caso van a considerar los expertos que han sido seleccionados por las partes e incluso decidir que haya un único experto que reciba instrucciones de ambas partes. ${ }^{29} \mathrm{Si}$ las partes no se pusieran de acuerdo en las instrucciones al experto, entonces sería el juez el encargado de decidir el objeto del peritaje, asumiendo que finalmente la prueba pericial tiene como objetivo brindar información relevante para la decisión judicial.

En todo caso, los jueces deberían observar con atención la información que se ha brindado a los peritos, ahí podrían encontrar explicaciones a los desacuerdos entre informes periciales e incluso adelantarse a ellos evitándolos en fase de admisión. Ello debería implementarse a través de una audiencia entre las partes, el juez y los expertos para dilucidar cuál debe ser la información brindada o incluso para delimitar de mejor manera los extremos del peritaje. En los sistemas de justicia actuales la situación prevista está muchas veces muy lejos de esto: a las partes se les pide, en ocasiones, simplemente que digan cuál sería el objeto del peritaje que proponen, para hacer luego una designación oficial del experto, con el que el juez no tendrá ningún contacto hasta que este le haga llegar su informe pericial. Quizá nos podríamos ahorrar muchos recursos de distinto tipo y sacar más provecho al experto si contáramos con su propia ayuda para establecer los extremos del peritaje y en ello participaran también ambas partes.

Lo anterior podría conllevar que las partes terminasen presentando conjuntamente un solo experto. Pero, entiéndase bien, no se trata solo de

28 Aunque no es el uso habitual, también podría decirse en este caso que el testimonio es parcial. El término "imparcialidad" pericial es un término complejo que puede tener distintos significados, al respecto puede verse Vázquez, 2018) y Dwyer (2008).

${ }^{29}$ Esta regla se encuentra vigente en el sistema inglés, véase la regla 35.7 de las Civil Procedure Rules y el numeral 7 de la Practice Direction 35. 
El diseño normativo de las pruebas periciales, a propósito del razonamiento...

incentivar que las partes se pongan de acuerdo en el nombramiento de un perito, a diferencia de lo que están haciendo muchos sistemas jurídicos, sino de que el experto participe en una reunión con las partes donde debata el objeto del peritaje y la información relevante que debe considerar. ${ }^{30}$

Dicho lo anterior, no basta con preocuparnos por los extremos del peritaje, hay que tomar en consideración la fiabilidad de los métodos, técnicas, etc., que empleará o ha empleado el experto. En esa línea, a partir de la experiencia estadounidense con la llamada trilogía Daubert, ${ }^{31}$ se ha planteado que todos los sistemas jurídicos deberían tener una admisibilidad más exigente para las pruebas periciales a efectos de evitar que entre al proceso conocimiento de baja o nula calidad. Por supuesto, ello supondría que el legislador correspondiente establezca claramente en el código procesal qué criterios jurídicos deberán considerar los jueces en adelante; pero, mientras eso no pase, debemos ver de qué manera podemos avanzar en la tarea con las herramientas con las que ya contamos, como el criterio de la relevancia o pertinencia previsto en todos nuestros ordenamientos. Y es que, en mi opinión, solo puede considerarse relevante aquel conocimiento experto cuya fiabilidad sea susceptible de conocerse por quienes tienen que decidir y, si es así, entonces las partes deben argumentarlo desde que ofrecen la prueba. Efectivamente, si entendemos que un elemento de juicio es relevante si, y solo si, permite fundar en él (por si solo o conjuntamente con otros elementos) una conclusión sobre la verdad del enunciado fáctico a probar, entonces, ¿ ¿ómo podríamos fundar una decisión sobre información cuya fiabilidad no se ha argumentado adecuadamente?

$\mathrm{Y}$ en este punto nos topamos con un gran problema en el uso de al menos parte del conocimiento experto que se utiliza en el proceso judicial: desconocemos su fiabilidad y desconocemos que desconocemos su

${ }^{30}$ Hay que lograr un balance entre los incentivos y desincentivos para acudir al litigio. Si los incentivos para acudir son grandes, entonces, el sistema adversarial y la oralidad se ponen en riesgo puesto que ambos son inherentemente time-consuming y caros. Hay que tomar en cuenta: los costos, los retrasos (delay) y que pueden hacerse públicas cuestiones que se hubiere preferido guardar en la privacidad, los malos ratos que se pueden pasar en tribunales, etc.

${ }^{31}$ Sobre esa parte de la experiencia estadounidense puede verse Vázquez (2016) y la literatura ahí citada. 
fiabilidad. Métodos y técnicas que han sido tradicionalmente usados en los procesos judiciales, como la identificación de huellas dactilares o las pruebas caligráficas, no han sido sujetas a estudios controlados que muestren que: I. en condiciones adecuadas es posible prever que alcanzarán resultados consistentes en $\mathrm{X}$ número de veces, y II. que tienen la capacidad de establecer lo que pretende establecer. Es decir, no tenemos información empírica sobre su fiabilidad: el único test que hemos aplicado a la expertise es la propia expertise, esto es, la preocupación suele girar en torno a si el sujeto puede ser considerado "realmente" un experto, si tiene la formación o habilidades correspondientes, si tiene los conocimientos suficientes, etc. Ninguno de dichos elementos provee información sobre cuán fiable es la técnica o el método usado por el experto y cuáles son las limitaciones del mismo, sus fuentes de error y grados de error.

En la mayoría de los procesos judiciales, cuando bien nos va, los expertos explican cómo supuestamente funcionan sus métodos o técnicas, pero hay muy poca información empírica sobre el funcionamiento real de los mismos. Uno de los mayores esfuerzos que se hicieron al respecto puede verse en el reporte "Strengthening Forensic Science in the United States: A Path Forward" realizado por el National Research Council of the National Academies y que, como su título indica, está dedicado al análisis de las ciencias forenses en el contexto estadounidense. En él se denuncia que, con excepción del ADN, ${ }^{32}$ no solo hay una falta de estudios controlados y/o otras pruebas que establezcan la fiabilidad de muchas de las ciencias forenses usadas cotidianamente en los procesos judiciales de los Estados Unidos, sino que además hay una tremenda divergencia en los tipos de titulación, ${ }^{33}$ certificación, formación, protocolos y metodologías empleadas por aquellos que llevan a cabo tales pruebas y participan como peritos en los juicios. Ello, por supuesto, dificulta la correcta identificación de los

\footnotetext{
32 Esta excepción no implica que las pruebas genéticas estén exentas de problemas. Sobre los diversos problemas que podrían encontrarse específicamente en las pruebas de $\mathrm{ADN}$ puede verse Murphy (2015).

${ }^{33}$ Esta situación no es propia de Estados Unidos, en España, por ejemplo, recientemente se ha llamado la atención sobre la importancia del título oficial del perito criminalístico nombrado por el juez. En ese sentido, véase Lucena, Franco, Iglesias, Pombar, y García Corrochano (2018).
} 
El diseño normativo de las pruebas periciales, a propósito del razonamiento...

delincuentes y pone en riesgo la corrección de las decisiones, aumentando los riesgos de las condenas falsas. ${ }^{34}$

¿Qué puede hacer el sistema jurídico para avanzar en el paso prioritario de contar con generalizaciones expertas bien soportadas por información empírica contrastada? Desde mi punto de vista, se deberían hacer dos labores, una vez que se ha tomado consciencia de su falta: exigir la inversión de mayores recursos económicos para la ciencia aplicada de utilización forense; y acudir a las comunidades expertas, pues es imposible que dicha información sea generada por expertos individuales. Los puentes que hasta ahora se han tendido entre la comunidad jurídica y la comunidad científica se han limitado la inmensa mayoría de las veces a que la primera le solicite a esta el envío de listados de expertos dispuestos a fungir como peritos, lo que es un acercamiento no sustantivo. Por el contrario, lo que deberíamos hacer es acercarnos para pedir detalles sobre cómo validan su conocimiento y cuáles son los resultados de dicha validación; y, en caso de que no se cuente con ese tipo de información, saber las razones de tal situación para evaluar cómo el sistema jurídico tiene que proceder. ${ }^{35}$

Todo lo anterior, por sí mismo, no resolverá los problemas de la prueba pericial, debe acompañarse de una adecuada formación de jueces y abogados. Tal como afirma Edmond, "para que las reformas procesales puedan lograr los objetivos deseados, es necesario que haya un cambio en la cultura y en los niveles de sofisticación técnica de los abogados y los jueces. Los abogados y los jueces deben entender por qué las prácticas tradicionales son inadecuadas y ser capaces y estar dispuestos a cambiarlas" (Edmond, 2012, p. 30, la traducción es mía). En otras palabras, los operadores jurídicos deben ser capaces de entender la necesidad de someter a las pruebas periciales a una sana crítica, pero aún más, deben entender que ello supone comprender adecuadamente el razonamiento pericial y no exclusivamente valorar al sujeto que lo dice o la forma en que lo dice a través de su informe.

34 El Innocence Project ya ha puesto sobre la mesa incluso algunos números que muestran en la realidad estas terribles consecuencias, véase los datos en https://www.innocenceproject.org/causes/misapplication-forensic-science/. Sobre este punto también llama la atención Duce (2018).

35 Por ejemplo, una de las opciones sería excluir sistemáticamente las pericias que se basen en métodos o técnicas cuya fiabilidad desconocemos. Vale la pena recalcar el aspecto social del desconocimiento en juego. 
Una vez que los operadores jurídicos son conscientes de ello, entonces corresponde ofrecerles un diseño normativo acorde a las exigencias.

\section{Conclusiones}

Los jueces deben valorar el razonamiento inferencial realizado por los peritos, sin embargo, la construcción de nuestros diseños normativos y de muchas de nuestras prácticas han apuntado a que los jueces se centren en valorar cuestiones diversas a ello. Como consecuencia, tenemos decisiones judiciales con conocimiento experto que no están justificadas. Es indispensable hacer un profundo cambio en dichas prácticas, un cambio que supone muy diversas líneas de acción, desde la educación en razonamiento probatorio para los operadores jurídicos, hasta la modificación de las herramientas que el sistema jurídico les ofrece para afrontar los grandes desafíos que su tarea exige. Tanto los jueces como los abogados y los fiscales parecen tener deficiencias importantes de información relevante a la hora de afrontar las pruebas periciales, pero no estamos ante deficiencias cognitivas insalvables. Hay mucha información empírica que pone sobre la mesa serios problemas en el uso de conocimiento experto, pero la manera en la que pueden afrontarse no es simplemente cambiando de sujeto, poniendo jueces expertos o comisiones de expertos que decidan las controversias jurídicas, sino empoderando a los actores para que cada uno desde su trinchera sea capaz de usar de manera racional conocimiento experto fiable.

\section{Bibliografía}

Cohen, L. J. (1983). Freedom of Proof. En Twinning, W. (ed.), Facts in Law (pp. 1-21), Wiesbaden: Franz Steiner Verlag GMBH.

Denyer, R. (2012). Case Management in Criminal Trials. Inglaterra: Hart Publishing.

Devis Echandía, H. (1972). Cientificidad de la prueba, en relación principalmente con los dictámenes periciales y la libertad de apreciación del juzgador. Revista de Derecho Procesal Iberoamericana, 1, 41-66. 
El diseño normativo de las pruebas periciales, a propósito del razonamiento...

Duce, M. (2018). Una aproximación empírica al uso y prácticas de la prueba pericial en el proceso penal chileno a la luz de su impacto en los errores del sistema. Política criminal, 13(25), 42-103.

Dwyer, D. (2008). Judicial Assessment of Expert Evidence. Cambridge: Cambridge University Press.

Edmond, G. (2012). Is Reliability Sufficient? The Law Commission and Expert Evidence in International and Interdisciplinary Perspective: Part 1. International Journal of Evidence \& Proof, 16(1), 30-65.

Ferrer, J. (2007). Valoración racional de la prueba. Madrid-Barcelona: Marcial Pons.

Freckelton, I., Goodman-Delahunty., J., Horan, J. y McKimmie, B. (2016). Expert Evidence and Criminal Jury Trials. Oxford: Oxford University Press.

Glaser, W. (1968). Pretrial Discovery and the Adversary System. New York: Russell Sage Foundation.

Goldman, A. (1991). Epistemic Paternalism: Communication Control in Law and Society. Journal of Philosophy, 88(3), 113-131.

Harker, D. (2015). Creating Scientific Controversies. Cambridge: Cambridge University Press.

Hart, H. L. A (1966). Law, Liberty and Morality. Stanford: Stanford University Press.

Hazel, G. (2013). Getting to the truth: experts and judges in the "hot tub", Civil Justice Quarterly, 32, 275-299.

Lackey, J. (2008). Learning from Words. Testimony as a Source of Knowledge. Oxford: Oxford University Press.

Lucena Molina, J. J., Franco Rodríguez, J. C., Iglesias García, M. J., Pombar Crespo, F. J. y García Corrochano, C. (2018). La relevancia del título oficial del perito criminalístico nombrado por el juez en la jurisdicción penal española. Madrid: Dykson.

Mnookin, J. (2006). Bifurcation and the Law of Evidence. University of Pennsylvania Law Review Pennumbra, 155, 134-145.

Movin Østergaar, J. (2016). An assessor on the tribunal: how a court is to decide when experts disagree. Civil Justice Quarterly, 35(4), 319-341.

Murphy, E. (2015). Inside the Cell: The Dark Side of Forensic DNA. New York: Nation Books. 
Schauer, F. (2006). On the Supposed Jury-Dependence of Evidence Law. University of Pennsylvania Law Review, 155, 165-202.

Schauer, F. y Spellman, B. (2013). Is Expert Evidence Really Different? Notre Dame Law Review, 89(1), 1-26.

Seidman, S. y Lempert, R. (2018). When Law Calls, Does Science Answer? A Survey of Distinguished Scientists \& Engineers. Daedalus, 147(4), 41-60.

Stafford, T., Holroyd, J. y Robin, S. (2018). Confronting Bias in Judging: A Framework for Addressing Psychological Biases in Decision Making. Manuscript.

Stein, F. (1990). El conocimiento privado del juez. Madrid: Editorial Centro de Estudios Ramón Areces.

Tonini, P. (2003). Prova scientifica e contraddittorio. Diritto penale e processo, 12, 1459-1465.

Tonini, P. (2011). Dalla perizia 'prova neutra' al contraddittorio sulla scienza. Diritto penale e processo, 3, 360-369.

van Rhee, C. H. (2007). Judicial Case Management and Efficiency in Civil Litigation, Cambridge: Intersentia.

Vázquez, C. (2015). De la prueba científica a la prueba pericial. MadridBarcelona: Marcial Pons.

Vázquez, C. (2016). La prueba pericial en la experiencia estadounidense. El caso Daubert. Jueces para la democracia, 86, 92-112.

Vázquez, C. (2018). La im/parcialidad pericial y otras cuestiones afines, Isonomía, 48, 69-107.

Vázquez, C. (en prensa). Los peritos de designación judicial a propósito del caso español. Los jueces, los grupos de expertos y el contexto procesal. En J. Ferrer Beltrán y C. Vázquez (eds.), El razonamiento probatorio en el proceso judicial, Madrid-Barcelona: Marcial Pons. 